\title{
Auras in patients with temporal lobe epilepsy and mesial temporal sclerosis.
}

\author{
Ali Akbar Asadi-Pooya \\ Thomas Jefferson University \\ Maromi Nei \\ Thomas Jefferson University \\ Ashwini Sharan \\ Thomas Jefferson University \\ Michael R. Sperling \\ Thomas Jefferson University
}

Follow this and additional works at: https://jdc.jefferson.edu/neurologyfp

Part of the Neurology Commons

Let us know how access to this document benefits you

\section{Recommended Citation}

Asadi-Pooya, Ali Akbar; Nei, Maromi; Sharan, Ashwini; and Sperling, Michael R., "Auras in patients with temporal lobe epilepsy and mesial temporal sclerosis." (2016). Department of Neurology Faculty Papers. Paper 108.

https://jdc.jefferson.edu/neurologyfp/108

This Article is brought to you for free and open access by the Jefferson Digital Commons. The Jefferson Digital Commons is a service of Thomas Jefferson University's Center for Teaching and Learning (CTL). The Commons is a showcase for Jefferson books and journals, peer-reviewed scholarly publications, unique historical collections from the University archives, and teaching tools. The Jefferson Digital Commons allows researchers and interested readers anywhere in the world to learn about and keep up to date with Jefferson scholarship. This article has been accepted for inclusion in Department of Neurology Faculty Papers by an authorized administrator of the Jefferson Digital Commons. For more information, please contact: JeffersonDigitalCommons@jefferson.edu. 


\title{
Brief Communication
}

Title: Auras in patients with temporal lobe epilepsy and mesial temporal sclerosis.

\author{
Ali A. Asadi-Pooya, M.D., Maromi Nei, M.D., Ashwini Sharan, M.D., Michael R. Sperling, \\ M.D. \\ Jefferson Comprehensive Epilepsy Center, Department of Neurology, Thomas Jefferson \\ University, Philadelphia, Pennsylvania, U.S.A.
}

\section{Address for correspondence:}

Ali A. Asadi-Pooya, M.D.

Department of Neurology

901 Walnut Street, Suite 435

Philadelphia, PA 19107

Phone: 215-955-1222

Fax: 215-955-3745

E-mails: aliasadipooya@yahoo.com; maromi.nei@jefferson.edu; ashwini.sharan@ jefferson.edu; michael.sperling@jefferson.edu

Key words: Aura; Surgery; Temporal lobe epilepsy.

Running title: Auras in mesial temporal sclerosis.

Word count for the paper: 1994. Word count for the abstract: 186 . Number of pages: 8 .

Number of references: 13. Characters in the title: 75; Number of tables: 1; Number of figures: 0. 


\begin{abstract}
:
We investigated auras in patients with drug-resistant temporal lobe epilepsy (TLE) and mesial temporal sclerosis (MTS). We also investigated the clinical differences between patients with MTS and abdominal auras and those with MTS and non-mesial temporal auras. All patients with drug-resistant TLE and unilateral MTS who underwent epilepsy surgery at Jefferson Comprehensive Epilepsy Center from 1986 through 2014 were evaluated. Patients with good postoperative seizure outcome were investigated. One hundred forty-nine patients (71 males and 78 females) were studied. Thirty-one patients (20.8\%) reported no auras, while 29 patients (19.5\%) reported abdominal aura, and 30 patients (20.1\%) reported non-mesial temporal auras; 16 patients $(10.7 \%)$ had sensory auras, 11 patients $(7.4 \%)$ had auditory auras, and five patients (3.4\%) reported visual auras. A history of preoperative tonic-clonic seizures was strongly associated with non-mesial temporal auras (odds ratio 3.8; 95\% CI: 1.15-12.98; $\mathrm{p}=0.02$ ). About one-fifth of patients who had MTS in their MRI and responded well to surgery reported auras that are historically associated with non-mesial temporal structures. However, the presence of presumed non-mesial temporal auras in a patient with MTS may herald a more widespread epileptogenic zone.
\end{abstract}

Key words: Aura; Surgery; Temporal lobe epilepsy. 


\section{Introduction}

Aura is a subjective ictal event that may precede a seizure and constitute a cardinal feature of focal epilepsies including temporal lobe epilepsy (TLE) [1]. Mesial temporal sclerosis (MTS) is the most common pathological abnormality associated with TLE [2, 3]. No clinical information or diagnostic method directly and definitively assesses the location and extent of the epileptogenic zone in focal epilepsies. However, concordance of multiple types of clinical data helps form a hypothesis regarding the localization of the epileptogenic zone. As the initial ictal symptoms, auras can provide important localizing and lateralizing information useful in determining the location of the epileptogenic zone [4]. Historically, some types of auras have been associated with TLE (e.g., abdominal auras, affective auras, and mnemonic auras), while some others (e.g., sensory auras) have been associated with non-temporal epilepsies [4]. In previous studies, abdominal auras have often been associated with mesial temporal lobe epilepsy [5-7]; however, other types of auras may also occur in these patients [5].

In this study, we investigated the types of auras in patients with drug-resistant TLE and unilateral MTS, who responded well to surgery. Rational for excluding the patients who failed to respond to surgery was to have a very uniform group of patients who preoperatively had MRIMTS and postoperatively responded well to the designed therapy with respect to seizure outcome. Response to appropriate treatment (i.e., standard anterior temporal lobectomy) was an indirect evidence for accuracy of the clinical diagnosis and also decreases the possibility of other variants of TLE such as temporal plus epilepsies [8], at least to some extent. We also investigated the potential clinical differences between patients with MTS and abdominal auras and those with MTS and non-mesial temporal auras (i.e., sensory, auditory or visual auras); to our knowledge, this comparison has never been done before. Such information may lead to a 
better understanding of the clinical manifestations of MTS. This may ultimately lead to a better understanding of the pathophysiology of MTS-TLE; for example, by providing the background for future network studies (e.g., fMRI studies).

\section{Methods}

In this retrospective study, all patients with a clinical diagnosis of drug-resistant TLE and unilateral MTS who underwent successful temporal lobectomy for epilepsy at Jefferson Comprehensive Epilepsy Center were recruited. Patients were prospectively registered in a database from 1986 through 2014. The diagnosis of MTS-TLE was based on clinical grounds (history and semiology), electroencephalographic (EEG) findings, and imaging [magnetic resonance imaging (MRI)]. There was no age limit to enter the study. For all patients, a comprehensive presurgical evaluation including a 1.5 Tesla brain MRI (epilepsy protocol) was performed. Magnetic resonance imaging studies were analyzed by neurologists and neuroradiologists with expertise in epilepsy. We classified patients as having MTS if they had clear signs of mesial temporal atrophy and/or sclerosis in their MRI. Patients with normal MRI and those with bilateral MTS or dual pathology and also, patients with concomitant psychogenic nonepileptic seizures were excluded from this study. Pathology specimens were reviewed by pathologists, but most results were not sufficient to provide a precise pathological classification.

All patients had a standard anterior temporal lobectomy. The patients were followed for up to five years after surgery (in many patients the data was missing beyond five years of followup). Seizure outcome was monitored periodically by office visits (by the treating physician), telephone contacts, and letters (by the nurse practitioners). Patients with postoperative seizure freedom for at least one year before their last visit were studied. Aura was not considered as a 
relapse; only postoperative tonic-clonic seizures and complex partial seizures were considered as relapse.

Age, gender, age at seizure onset (i.e., the first afebrile seizure), seizure type(s), MRI findings, date of surgery, date of the first relapse (if any) and date of the last contact with all patients were registered routinely. Source of information about auras was the history obtained by the board certified neurologists (epileptologists). Six groups of patients were identified, according to their preoperative auras. The first group included patients with abdominal aura (abdominal discomfort including nausea, emptiness, tightness, butterflies, malaise, pain, or hunger; sensation may rise to chest or throat); the second group of patients reported affective auras (i.e., fear, anxiety, depression, joy, or anger); the third group reported mnemonic auras [i.e., feelings of familiarity (déjà-vu) or unfamiliarity (jamais-vu)]; the fourth group had nonmesial temporal auras (i.e., sensory, auditory and visual); the fifth group reported other types of auras (e.g., dizziness, strange feelings, etc.); and finally, the last group did not report an aura preceding their seizures.

Demographic variables and relevant clinical variables were summarized descriptively to characterize the study population. Patients with non-mesial temporal auras (i.e., sensory, auditory or visual auras) were compared with patients who had abdominal auras. Pearson Chi-Square, ttest, and Kolmogorov-Smirnov test were used for statistical analyses. P value less than 0.05 was considered as significant. Variables that were significant or nearly so in univariate tests were assessed in a logistic regression model. Odds ratio and 95\% confidence interval (CI) were calculated. This study was conducted with the approval by Thomas Jefferson University Institutional Review Board. 


\section{Results}

During the study period, 794 patients had temporal lobe surgery for drug-resistant temporal lobe epilepsy. Magnetic resonance imaging results were as follows: MTS in 306, dual pathology in 19, other findings in 278, normal results in 168, and missing results in 23 patients. Two-hundred and thirty-seven patients had complete data with regard to preoperative seizure information (i.e., aura types). Of these, 149 patients (62.8\%) reported no seizure for more than one year before their last visit and were studied. We included 71 men and 78 women. Age at epilepsy onset was $13.8 \pm 11.5$ years. Age at surgery was $36.5 \pm 11.2$ years. Thirty-one patients (20.8\%) reported no auras, while 29 patients $(19.5 \%)$ reported abdominal aura, $10(6.4 \%)$ had affective aura, 7 (4.7\%) reported mnemonic aura, 30 (20.1\%) reported non-mesial temporal auras and the remainder had other auras (e.g., indescribable feeling, dizziness, or headache). Among patients with non-mesial temporal auras, 16 patients $(10.7 \%)$ had sensory auras (eight patients as a single aura and eight as a part of multiple auras), 11 patients $(7.4 \%)$ had auditory auras (seven patients as a single aura and four as a part of multiple auras), and five patients (3.4\%) reported visual auras (two patients as a single aura and three as a part of multiple auras). Two patients had multiple non-mesial temporal auras (one had sensory and auditory and the other had visual and auditory auras). Patients with non-mesial temporal auras (30 patients) were compared with patients who had abdominal auras (29 patients) in Table 1. Negative history of childhood febrile seizures and positive history of preoperative tonic-clonic seizures were associated with nonmesial temporal auras in univariate analyses. We then performed a logistic regression analysis, assessing these two variables. A history of preoperative tonic-clonic seizures was strongly associated with non-mesial temporal auras compared with patients with abdominal auras (odds 
ratio $3.8 ; 95 \% \mathrm{CI}: 1.15-12.98 ; \mathrm{p}=0.02 ; 70.7 \%$ of the cases were correctly predicted by the model). History of childhood febrile seizures $(\mathrm{p}=0.08)$ was not a significant risk factor.

\section{Discussion}

As the first ictal symptoms, auras can provide important information useful in determining the location of the epileptogenic zone in patients with focal epilepsy. Abdominal auras include sensations of nausea, pain, or indescribable discomfort in the abdominal or periumbilical area that can be static, rise to the chest and throat, or descend into the lower abdominal region [4]. The seizure onset zone for abdominal auras may be the anterior insular cortex, frontal operculum, or mesial temporal structures [4]. However, abdominal auras have often been associated with mesial temporal lobe epilepsy [5-7]. In a previous study [7], presence of abdominal aura was a good prognostic indicator for seizure free outcome after temporal lobectomy in patients with MTS-TLE. On the other hand, it is not unusual to observe other types of auras in patients with MTS-TLE. Similarly, it is not rare to observe abdominal auras in other focal epilepsy syndromes [9]. In one previous study [5], abdominal auras were more frequent with TLE (117 of 223 patients, 52\%) than in extratemporal epilepsy (13 of 113 patients, 12\%, p $<0.0001$ ) and more frequent in mesial TLE (70 of 110 patients, 64\%) than in neocortical TLE (16 of 41 patients, 39\%, $\mathrm{p}=0.007$ ). In the current study, we observed that about one-fifth of the patients who had MTS in their MRI and responded well to standard anterior temporal lobectomy reported auras that are historically associated with non-mesial temporal structures (i.e., sensory, auditory or visual auras); one-fifth had abdominal auras and another one-fifth had no auras associated with their seizures. In a previous study [9], of 333 patients who had undergone temporal lobectomy for drug- resistant TLE, 26 (7.8\%) patients had somatosensory auras. These 
authors observed that [10], compared to their non-somatosensory auras counterparts, patients with somatosensory auras had the same clinical and imaging characteristics, but had a higher rate of breakthrough seizures, although most (54\%) were still able to achieve Engel class of I outcome after surgery [10]. In another study of 400 MTS-TLE patients [11], 12\% of the patients reported somatosensory auras; $10 \%$ had visual and 7\% reported auditory auras. This study also found that patients with different auras could have good surgery outcome [11]. These findings are similar to ours and indicate that in a patient with drug-resistant seizures and mesial temporal epilepsy, observation of a presumed non-mesial temporal aura should not deter from surgery as a potential curative option. However, absence of abdominal auras may herald a lower chance of seizure free outcome postoperatively in patients with MTS-TLE, as we observed in a previous study [7]. This information should be discussed with the patients in a preoperative counselling session.

Patients with MTS and non-mesial temporal auras (i.e., sensory, auditory or visual auras) had few, but significant clinical differences compared with those with abdominal auras; a history of preoperative tonic-clonic seizures was strongly associated with non-mesial temporal auras. Presence of the presumed non-mesial temporal auras in a patient with MRI-MTS may herald a more widespread epileptogenic zone compared with that in patients who report abdominal auras. Alternatively, this may suggest a different epileptic network and spread pattern compared with the network responsible for seizures in patients with abdominal aura. Our previous observation that patients with drug-resistant MTS-TLE, who reported abdominal auras preceding their seizures, fared better postoperatively with regard to seizure control compared to those with other types of auras [7] support the hypothesis that a more restricted epileptogenic zone is responsible for seizure generation in patients with abdominal auras. In addition, evidence obtained with 
fMRI suggests more widespread derangements in brain function and connectivity in TLE patients with tonic-clonic seizures (compared with those without this seizure type) [12], and we observed that a history of preoperative tonic-clonic seizures was strongly associated with nonmesial temporal auras. However, the hypothesis of various epileptic networks in patients with different types of auras, despite a similar core of pathology (i.e., mesial temporal sclerosis), could not be excluded. Future functional and structural connectivity studies and also precise network analyses may answer to these questions.

\section{Study limitations}

This was a retrospective study and patient data came from a single institution. Also, because this was a clinic-based series of the patients with drug-resistant MTS-TLE, they may not represent the full spectrum of MTS-TLEs. Finally, pathology specimens were reviewed by pathologists, but most results were not sufficient to provide a precise pathological classification according to the international recommendation for a comprehensive neuropathologic workup of epilepsy surgery brain tissue [13].

\section{Conflict of interest}

Ali A. Asadi-Pooya, M.D., reports no disclosures.

Maromi Nei, M.D., Research: Upsher-Smith Laboratories, NINDS.

Ashwini Sharan, M.D., SJM, Clinical Trial Site - Grant, consulting; Cerebral

Therapeutics - ownership interest; Tiger labs - ownership interest; Saluda Medical - Clinical Trial Site. 
Michael R. Sperling, M.D., Consulting: UCB Pharma; Research: contracts with Thomas

Jefferson University, Eisai, UCB Pharma, Sunovion, SK Life Sciences, Marinus, Lundbeck, Medtronics, Visualase, Accorda, Upsher-Smith, Brain Sentinel.

\section{Acknowledgment}

This was a non-funded study. 


\section{Reference}

[1] Blume WT, Lüders HO, Mizrahi E, et al. Glossary of descriptive terminology for ictal semiology: report of the ILAE task force on classification and terminology. Epilepsia 42(9) (2001) 1212-1218.

[2] Téllez-Zenteno JF, Hernández-Ronquillo L. A review of the epidemiology of temporal lobe epilepsy. Epilepsy Res. Treat. 2012 (2012) 630853.

[3] Babb TL, Brown WJ. Pathological findings in epilepsy. In: Engel Jr., J. (Ed.), Surgical Treatment of the Epilepsies. Raven Press, New York, (1987) pp. 511-540.

[4] Foldvary-Schaefer N, Unnwongse K. Localizing and lateralizing features of auras and seizures. Epilepsy Behav. 20(2) (2011) 160-166.

[5] Henkel A, Noachtar S, Pfander M, et al. The localizing value of the abdominal aura and its evolution: a study in focal epilepsies. Neurology 58 (2002) 271-276.

[6] Duncan JS, Sagar HJ. Seizure characteristics, pathology, and outcome after temporal lobectomy. Neurology 37(3) (1987) 405-409.

[7] Asadi-Pooya AA, Nei M, Sharan A, Sperling MR. Type of preoperative aura may predict postsurgical outcome in patients with temporal lobe epilepsy and mesial temporal sclerosis. Epilepsy Behav. 50 (2015) 98-100.

[8] Barba C, Rheims S, Minotti L, et al.Temporal plus epilepsy is a major determinant of temporal lobe surgery failures. Brain (2015) Dec 22. pii: awv372. [Epub ahead of print].

[9] Wieser HG; ILAE Commission on Neurosurgery of Epilepsy. ILAE Commission Report. Mesial temporal lobe epilepsy with hippocampal sclerosis. Epilepsia 45(6) (2004) 695-714. [10] Perven G, Yardi R, Bulacio J, et al. The relevance of somatosensory auras in refractory temporal lobe epilepsies. Epilepsia 56(10) (2015) e143-8. 
[11] Dupont S, Samson Y, Nguyen-Michel VH, et al. Are auras a reliable clinical indicator in medial temporal lobe epilepsy with hippocampal sclerosis? Eur. J. Neurol. 22(9) (2015) 1310-6. [12] He X, Doucet G, Sperling MR, Sharan A, Tracy J. Reduced thalamocortical functional connectivity in temporal lobe epilepsy. Epilepsia 56(10) (2015) 1571-9.

[13] Blümcke I, Aronica E, Miyata H, et al. International recommendation for a comprehensive neuropathologic workup of epilepsy surgery brain tissue: A consensus Task Force report from the ILAE Commission on Diagnostic Methods. Epilepsia (2016) doi: 10.1111/epi.13319. [Epub ahead of print]. 
Table 1. Patients with mesial temporal sclerosis and non-mesial temporal auras compared with patients who had abdominal auras.

\begin{tabular}{|c|c|c|c|}
\hline Variables & $\begin{array}{l}\text { Patients with non-mesial } \\
\text { temporal auras (30 } \\
\text { patients) }\end{array}$ & $\begin{array}{c}\text { Patients with } \\
\text { abdominal auras (29 } \\
\text { patients) }\end{array}$ & $\begin{array}{c}\text { p } \\
\text { value }\end{array}$ \\
\hline Gender (Male: Female) & $14: 16$ & $17: 12$ & 0.4 \\
\hline Age at epilepsy onset & $13.2 \pm 9.9$ years & $13.1 \pm 11.7$ years & 0.7 \\
\hline Duration of disease & $20.2 \pm 13$ years & $22.2 \pm 10.3$ years & 0.2 \\
\hline Childhood febrile seizure & 8 out of $28 *(28.6 \%)$ & 16 out of $29(55.2 \%)$ & 0.04 \\
\hline $\begin{array}{l}\text { Preoperative tonic-clonic } \\
\text { seizures }\end{array}$ & 18 out of $29 *(62.1 \%)$ & $11(37.9 \%)$ & 0.06 \\
\hline Family history of epilepsy & 6 out of $29 *(20.7 \%)$ & 12 out of $29(41.4 \%)$ & 0.1 \\
\hline $\begin{array}{l}\text { MRI laterality of the lesion } \\
\text { (Right : Left) }\end{array}$ & $14: 16$ & $16: 13$ & 0.6 \\
\hline $\begin{array}{l}\text { Posterior temporal or } \\
\text { occipital interictal } \\
\text { epileptiform discharges }\end{array}$ & 0 & 0 & -- \\
\hline $\begin{array}{l}\text { Intracranial EEG } \\
\text { monitoring }\end{array}$ & 3 & 0 & 0.08 \\
\hline
\end{tabular}

*Some data were missing. 